\title{
DEVELOPMENT OF A MULTI CAMERA CALIBRATION FOR AN ANALYSIS AND MANIPULATION SYSTEM OF BIOLOGICAL SAMPLES IN PETRI DISHES
}

\author{
Matthias Hardner ${ }^{1 *}$, Danilo Schneider ${ }^{1}$ \\ ${ }^{1}$ Institute of Photogrammetry and Remote Sensing, TU Dresden, Germany - (matthias.hardner, danilo.schneider)@tu-dresden.de
}

\section{Commission II}

KEY WORDS: calibration, multi-camera, robot tracking, automation

\begin{abstract}
:
Cameras can provide accurate 3D positioning for a robotic system. Calibration of interior orientation as well as the relative orientation when using multiple cameras is a necessary step. Integration of the automatic calibration process into the robot system is useful to provide a simple system for the user. The paper presents a method for calibrating a multi camera system using the open source Ceres solver and compares it to a commercial software. The motivation is a measurement system for laboratory automation where biological samples in Petri dishes will be manipulated with different tools. For this application we will examine the camera setup, show first results of the calibration and the possible accuracy. Lastly we will draw conclusions for the final system prototype and necessary improvements to be implemented in order to provide accurate measurements.
\end{abstract}

\section{INTRODUCTION}

Automation of repetitive tasks is used in more and more fields. One example of such a task in laboratories is the test for Legionella contamination while analyzing drinking water. Regular inspections result in a large number of samples to be analyzed constantly. Automation of this process is desirable, in particular for the task of extracting part of a culture from one Petri dish and placing it in a new one. Furthermore, automated analyses and robotic manipulation of biological samples is applicable to many other fields. Based on previous work (Vogel et al., 2015, Oberthür et al., 2016) the cooperative project 3Dbot focuses on constructing a demonstrator for these tasks.

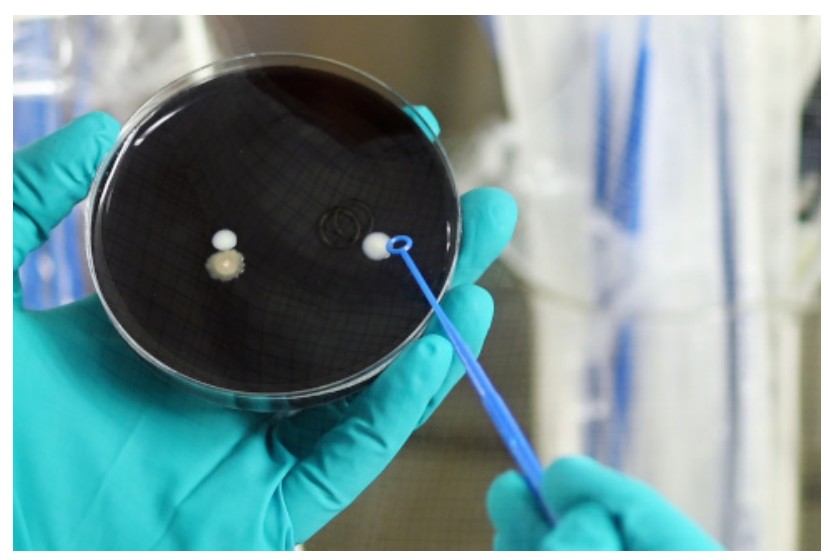

Figure 1. Manual collection of a sample for checking if it is a Legionella culture or something different.

The goal of the cooperative research project 3Dbot is the development of hardware and software for a demonstrator of a robot system to proof that automated sample analyses and manipulation is possible. The project specific task is the optical analysis of a Legionella sample, the extracting of a small portion and its following placement in a second Petri dish (Figure 1). Focus is

\footnotetext{
*Corresponding author
}

on a fully automated processing chain of samples for approximate 12 hours without any manual input. One crucial restriction is the final price of the measurement system to be low enough for laboratories to be considered. Therefore, low cost mechanical components for the robot where chosen with the idea of increasing the accuracy using photogrammetric methods.

Photogrammetry is able to support the measurement system at multiple tasks such as providing accurate $3 \mathrm{D}$ position information of mobile system elements and interest points on the samples as well as calculating a full 3D model of more complex biological samples. The paper focuses on the concept for the calibration of the multi camera system and will present challenges, first results and optimization potential.

\subsection{Related Work}

The challenge of the position feedback and accuracy of robot systems can be solved through the addition of cameras. One method is the kinematic calibration for the precise description of the robot system, often by attaching visible markers to the end effector (Zhang et al., 2017, Renaud et al., 2006). This solution is limited to parameters which are stable over time. One alternative method is the dynamic control and correction of the robot pose using cameras, which is often called "visual servoing". This approach is able to improve the position accuracy of low cost systems as well as of high quality industrial robots (Hefele, Brenner, 2001, Durovic et al., 2017). For smooth pose estimation the usage of a Kalmann filter is proposed in (Shu et al., 2018).

The required camera calibration is an extensively researched field in Photogrammetry as well as in computer vision. Different camera and distortion models and multiple methods for solving the systems can be used (Luhmann et al., 2016, Triggs et al., 1999). Multi camera calibration as a special case is often being used for the tracking of robots and measurements in factory environments (Werner et al., 2018, Sargeant et al., 2016). 


\subsection{System}

The system consists of a movable platform (X-direction), mounted on it are 4 cameras and a robot arm (Z-/Y-direction) for the manipulation of the samples (Figure 1). The platform can move over multiple work positions, that can each hold and move one Petri dish (rotate/tilt). Spherical markers are attached to the robot end effector as well as to the Petri dish mounting. Additional markers are added to the measurement system to track the position of the movable platform. The placement and removal of the Petri dishes is done by a separate robot, which will be not subject of this paper. System calibration will be based on the use of existing markers in the system and not an additional calibration field. These markers are also used for tracking the components, as well as calculating the system accuracy.

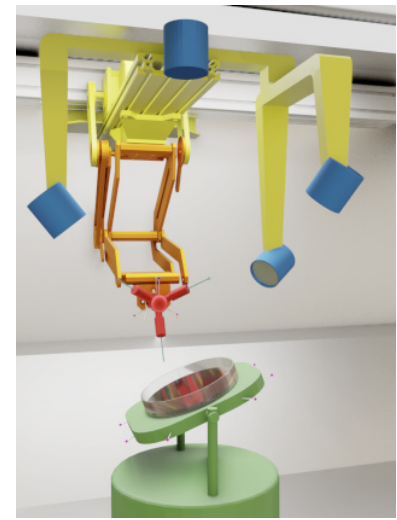

(a) System overview

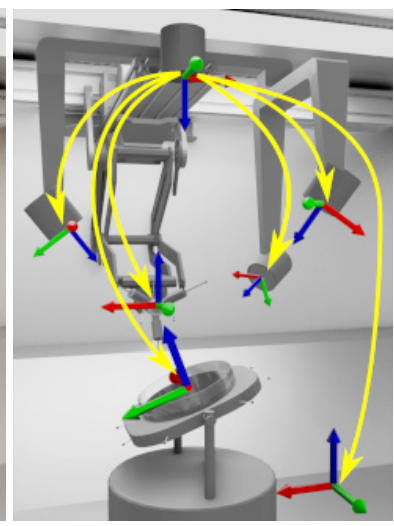

(b) Transformation overview
Figure 2. Schematic representation of the measurement system with the movable platform (yellow), the cameras (blue), the robot arm (orange) with the end effector (red) and the Petri dish mount (green). All transformations are described in relation to the primary camera.

\begin{tabular}{l|ll} 
& Primary & Secondary (3x) \\
\hline Camera & Basler acA4112- & Basler daA1600- \\
& 30uc & 60 um CS \\
Sensor size $[\mathrm{mm}]$ & $14,1 \times 10,3$ & $7,2 \times 5,4$ \\
Resolution $[\mathrm{px}]$ & $4096 \times 3000$ & $1600 \times 1200$ \\
Pixel pitch $[\mu \mathrm{m}]$ & 3.44 & 4.5 \\
Lens & Computar & Pentax H614- \\
& V1628-MPY2 & MQ \\
Focal length $[\mathrm{mm}]$ & 16 & 6 \\
Sensor type & RGB & Monochromatic
\end{tabular}

Table 1. Summary of camera and lens parameter.

The calibration of the camera system is a basic requirement for the system to perform accurate 3D measurements. The interior orientation (principal distance and principal point) as well as the distortion parameters of all four cameras have to be determined. Since all cameras are mounted together the relative orientation (6 parameters) for each of the secondary cameras to the primary camera is also calibrated. The tie points are located in three different reference frames (on the end effector, on the Petri dish mount and in the system itself), so the exterior orientation for the primary camera has to be calculated in all three systems. For the camera calibration the three components are moved one by one or all together to different locations within the system and are captured through all cameras. The unknown parameters of the calibration can divided in to two category's, the assumable time invariant and the time variant parameters.

\section{- Time invariant parameter:}

- Interior orientation (3 for each camera)

- Distortion (5 for each camera)

- Relative orientation (6 for each secondary camera)

- Object points (3 for each point)

\section{- Time variant parameter:}

- Exterior orientation of primary camera (6 for each time step and each component)

\subsection{Aim of Paper}

In the paper we will present the full calibration concept and first results of the implementation, a first accuracy assessment of the implemented method as well as a comparison to a multi camera calibration with a commercial software (Aicon 3D Studio). In contrast to the final system a separate calibration field with all points in one reference frame is used. The cameras were mounted in a similar configuration to the final measurement system (Figure 3).

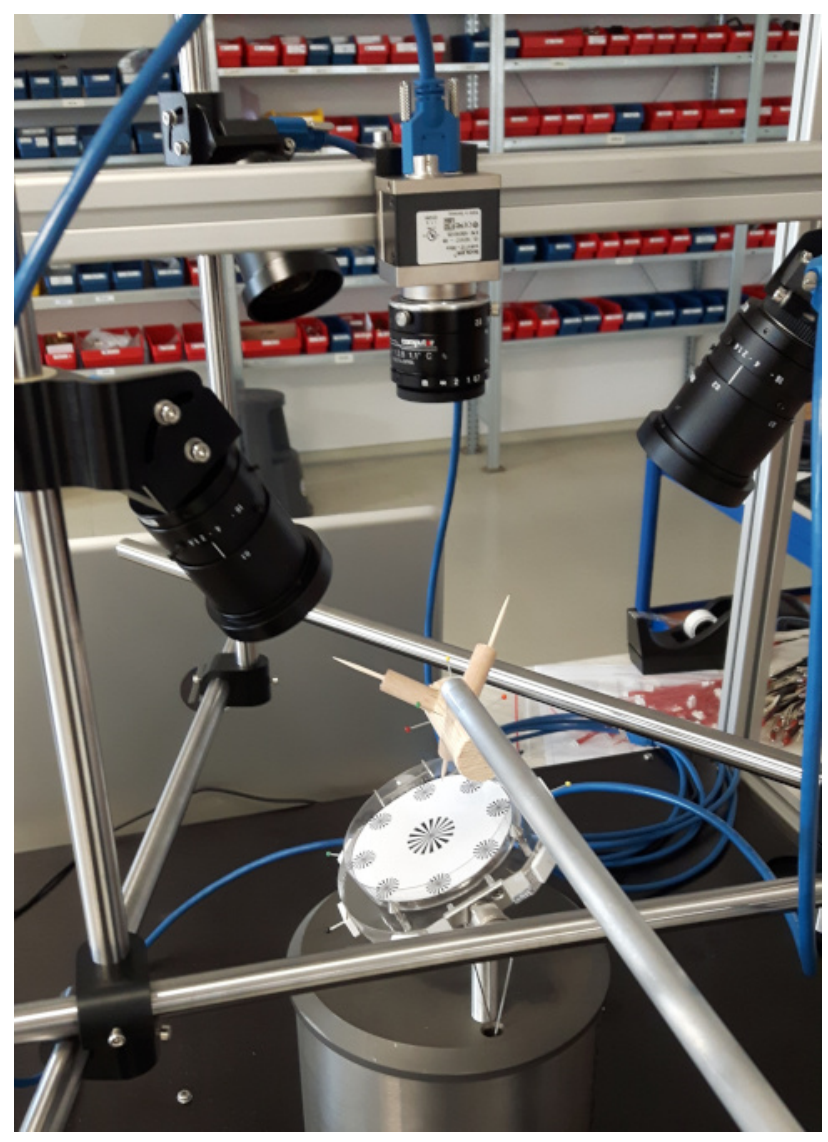

Figure 3. Prototype of the measurement system with the primary camera, the secondary cameras. For the calibration test the end effector and the Petri dish mount were removed for the use of the calibration field.

\section{METHOD}

The calibration is implemented using the Ceres solver for the bundle adjustment (Agarwal et al., 2019). Features of this opensource library are the simple definition of the functional model, automatic differentiation, different methods of solving the system. Advantages for our application are the availability for 


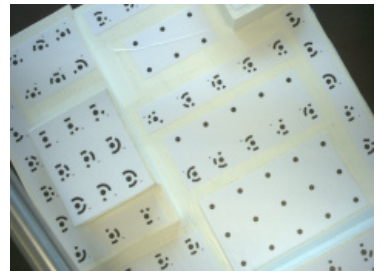

(a) Primary camera

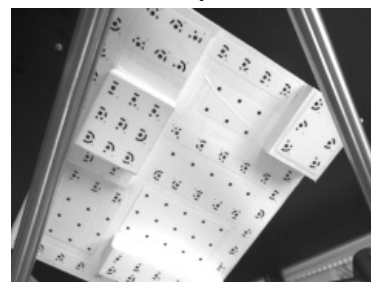

(c) Secondary Camera 2

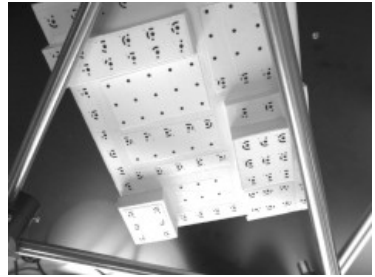

(b) Secondary Camera 1

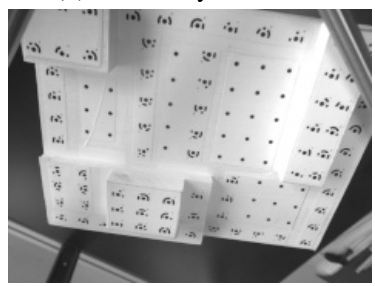

(d) Secondary Camera 3
Figure 4. Example images of all four cameras for one time step with the calibration field.

Linux and the already for performance optimized software. The library is also providing some of the necessary functions for evaluating the result which is important, since the final measurement system has to perform the calibration fully automatic.

\subsection{Model}

The functional Model is based on the pinhole camera model, with the principal distance $(c k)$, the principal point $\left(x_{0}, y_{0}\right)$, the radial-symmetric $(\mathrm{rad})$ and the tangential $(\tan )$ distortion parameters. The object points $(X, Y, Z)$ are projected to the image plane $\left(x^{\prime}, y^{\prime}\right)$ to the image using the rotation matrix $\left(r_{11}\right.$, ...) and the coordinates of the projection center $\left(X_{0}, Z_{0}, Z_{0},\right)$ of the exterior orientation.

$$
\begin{aligned}
x^{\prime} & =c k \frac{r_{11}\left(X-X_{0}\right)+r_{21}\left(Y-Y_{0}\right)+r_{31}\left(Z-Z_{0}\right)}{r_{13}\left(X-X_{0}\right)+r_{23}\left(Y-Y_{0}\right)+r_{33}\left(Z-Z_{0}\right)} \\
y^{\prime} & =c k \frac{r_{12}\left(X-X_{0}\right)+r_{22}\left(Y-Y_{0}\right)+r_{32}\left(Z-Z_{0}\right)}{r_{13}\left(X-X_{0}\right)+r_{23}\left(Y-Y_{0}\right)+r_{33}\left(Z-Z_{0}\right)}
\end{aligned}
$$

Distortion parameter are based on the Brown (Brown, 1971) with $R_{0}=0$ to ensure compatibility with OpenCV (Bradski, 2000) functions. Parameters of the radial-symmetric $\left(A_{1}, A_{2}\right.$, $\left.A_{3}\right)$ and the tangential $\left(B_{1}, B_{2}\right)$ lens distortion are considered.

$$
\begin{gathered}
r^{2}=x^{\prime 2}+y^{\prime 2} \\
\Delta r_{\text {rad }}^{\prime}=\left(\left(A_{3} r^{2}+A_{2}\right) r^{2}+A_{1}\right) r^{2} \\
\Delta x_{\text {tan }}^{\prime}=B_{1}\left(r^{2}+2 x^{\prime 2}\right)+2 B_{2} x^{\prime} y^{\prime} \\
\Delta y_{\text {tan }}^{\prime}=B_{2}\left(r^{2}+2 y^{\prime 2}\right)+2 B_{1} x^{\prime} y^{\prime}
\end{gathered}
$$

The distortion values and principle point $\left(x_{0}, y_{0}\right)$ is applied to get the final image point $(x, y)$.

$$
\begin{gathered}
x=x^{\prime}+x_{0}+x^{\prime} \Delta r_{r a d}^{\prime}+\Delta x_{t a n}^{\prime} \\
y=y^{\prime}+y_{0}+y^{\prime} \Delta r_{r a d}^{\prime}+\Delta y_{t a n}^{\prime}
\end{gathered}
$$

\subsection{Bundle Adjustment}

The model is added as a cost function to the bundle adjustment problem by defining it as a $\mathrm{C}++$ function template. Manual by hand, numeric or automatic differentiation can be used with the Ceres-solver. In this implementation the recommended automatic differentiation is chosen.

The influence of outlier observations caused by wrongly matched points or errors in marker detection are reduced by the use of the Huber loss function (Huber, 1964) with a value of 0.01 . The loss function will cause the cost to be linear for large image residuals but quadratic for small residuals. The bundle is solved using the Levenberg-Marquardt algorithm.

If object point coordinates are adjusted in the calibration with the other parameters additional, constraints for a free network adjustment are necessary to avoid rank deficiency. Adding these seven parameter constraints is only possible using additional cost functions. For each coordinate system a constraint is added where the approximated object points are used as observations for a transformation of the constraints with the object points. This will define the datum by fitting it to the approximated object point coordinates.

The implementation allows the configuration of the calibration using one XML - File. Camera parameter, object points, coordinate systems and options for bundle adjustment can be set. Additionally every of the parameters can be fixed, which allows the re-calibration of selected parameters (for example the principal point of one camera).

\section{RESULTS}

The points coordinates of the calibration field were determined beforehand using two calibrated scale bars. The calibration field is moved to different locations and is captured with all cameras. In contrast to the final system all points are in one coordinate system to allow calibration in Aicon 3D Studio as the reference. The image coordinates from Aicon are also used as observations in our implementation using Ceres.

\section{Testing procedure:}

1. 3D - calibration field captured by all four cameras over 40 time steps (160 images)

2. Marker detection in Aicon 3D Studio

3. Multi-camera calibration in Aicon 3D Studio

4. Multi-camera calibration with Ceres using image coordinates from Aicon (as outlier marked observations were not imported)

\begin{tabular}{lr}
\hline Unknown time invariant parameter & \\
Interior orientation (3 per camera) & 12 \\
Distortion parameter (5 per camera) & 20 \\
Relative orientation (6 per secondary camera) & 18 \\
\hline Unknown variant parameter & \\
Exterior orientation primary camera (6 per time step) & 240 \\
\hline Fixed known parameter & \\
Object points (3 per point) & 363 \\
\hline Observations & \\
Image points (2 per point) & 18596 \\
\hline
\end{tabular}

Table 2. Overview over parameters and observations. 
In the following sections we compare the results of the Ceres multi calibration to the reference of Aicon and look at some remaining systematic errors. Afterwards the results of the Ceres method are analyzed with respect to the 3Dbot project and its requirements.

\subsection{Comparison to reference calibration}

To determine the accuracy of the multi camera calibration in Ceres we compare it to the results in Aicon 3D Studio. Object points where fixed, while interior, relative and exterior orientation where all adjusted over the 40 time steps.

The resulting calibration parameters (Table 4) show small differences between the Ceres and Aicon. The primary camera has the largest differences at the principal distance with $\Delta c k=$ $0.013 \mathrm{~mm}$ and the principal point $\left(\Delta y_{0}=0.0064 \mathrm{~mm}=1.86\right.$ Pixel).

When comparing image residuals of between the two calibrations only small difference are visible. Splitting the image residuals by cameras a significant difference is visible between the primary and secondary cameras (Figure 5, Table 3). While the secondary cameras have a larger pixel size than the primary camera the interpolation due to the Bayer pattern could be a reason for the differences. Further reasons could be a better description of the secondary cameras by camera model or less accurate marker detection in the high resolution color images.

\begin{tabular}{l|rr|rr} 
& \multicolumn{2}{|c|}{ Ceres } & \multicolumn{2}{c}{ Aicon } \\
& $\mathrm{x}$ & $\mathrm{y}$ & $\mathrm{x}$ & $\mathrm{y}$ \\
\hline Primary camera & 6.28 & 7.38 & 6.22 & 7.16 \\
Secondary camera 1 & 3.29 & 3.94 & 3.32 & 3.96 \\
Secondary camera 2 & 3.39 & 3.90 & 3.41 & 3.99 \\
Secondary camera 3 & 3.10 & 3.93 & 3.12 & 4.01
\end{tabular}

Table 3. RMSE of the image residuals in $\mu m$ for Ceres and the reference of each camera.

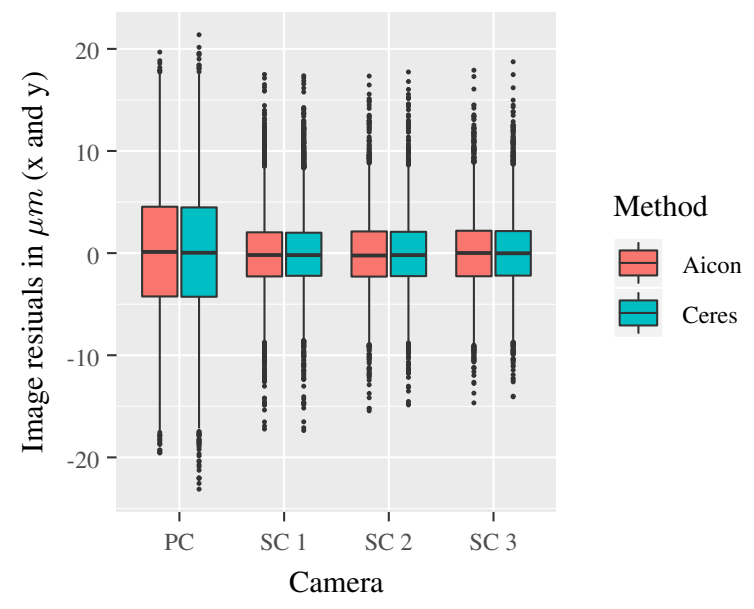

Figure 5. Image residuals ( $\mathrm{x}$ and $\mathrm{y}$ ) split by camera.

\subsection{Systematic Errors}

Plots of the residuals for one single time step show some remaining systematic errors (Figure 6) left. Image residuals of Aicon calibration show similar problems. Adjusting the 3D points during the calibration as well as adjusting the relative orientation for each time step reduces these systematic errors only slightly. Further reasons for this have to be investigated when the final prototype including the markers in different coordinate systems is build.

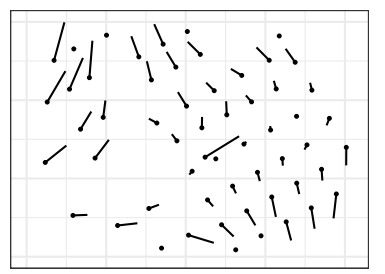

(a) Primary camera

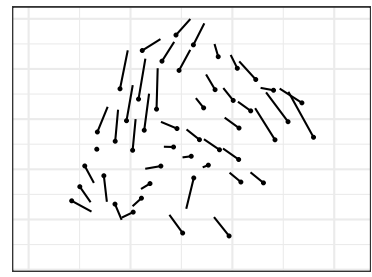

(c) Secondary camera 2

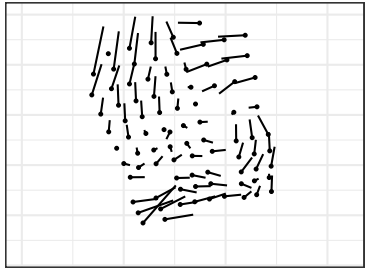

(b) Secondary camera 1

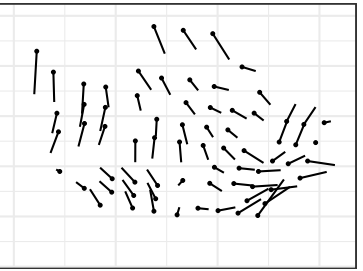

(d) Secondary camera 3
Figure 6. Example plot of image residuals after Ceres calibration for one time step show systematic errors (images see Figure 4).

\subsection{Scale errors}

One important question is, how accurate $3 \mathrm{D}$ points can be measured in the camera system using the four images of one time step. The accuracy in object space is determined by calculating the distance error between 31 evenly distributed point pairs on the calibration field. First the 3D coordinate is triangulated and then the points are adjusted using observation of all cameras. The known length between the points is taken as the reference. The errors where computed over all time steps. Since the points are not always visible in all four cameras the adjusted coordinates use between 4 and 8 image points (each with $\mathrm{x}$ and $\mathrm{y}$ ). The RMSE for all the distance measurements is $0.17 \mathrm{~mm}$. When only considering measurements with more than 6 observed image points the RMSE drops to $0.15 \mathrm{~mm}$ (Table 5).

\begin{tabular}{l|rr} 
Image points used & $4-6$ & $7-8$ \\
\hline RMSE [mm] & 0.172 & 0.146 \\
Max [mm] & 0.715 & 0.363 \\
Min [mm] & -0.567 & -0.433
\end{tabular}

Table 5. Statistics over distance error measurements by the number of image points used.

\subsection{Orientation errors}

Since the number of markers at the end effector and the Petri dish mount are limited a interesting question is the possible positioning accuracy of a system component in relation to the number of points. First multiple subsets of points where chosen. For each set the exterior orientation of the main camera was adjusted using observations of all cameras. Relative and Interior orientation as well as object point coordinates were fixed. The position calculated with the limited number of points was compared to a position using all available points. Note that the points where located around the coordinate system origin of the component, so rotational errors have a low influence on the result.

As can be seen in Figure 7, 16 observed image points for one coordinate system are necessary to determine the position of the system component within max deviations of around $\pm 0.20 \mathrm{~mm}$ in both the X- and Y- direction with a RMSE of $0.049 \mathrm{~mm}(\mathrm{X})$ 


\begin{tabular}{|c|c|c|c|c|c|c|c|c|}
\hline & \multicolumn{2}{|c|}{ Primary Camera } & \multicolumn{2}{|c|}{ Secondary Camera 1} & \multicolumn{2}{|c|}{ Secondary Camera 2} & \multicolumn{2}{|c|}{ Secondary Camera 3} \\
\hline & Ceres & Aicon & Ceres & Aicon & Ceres & Aicon & Ceres & Aicon \\
\hline$c k[\mathrm{~mm}]$ & -16.9198 & -16.9324 & -6.3379 & 5.3408 & -6.3140 & -6.3154 & -6.3351 & -6.3378 \\
\hline$x_{0}[1$ & 7 & 4.7 & -02 & -4 & 8.6 & 8. & -1 & -1.0 \\
\hline$y_{0}[1$ & $-1.42 \mathrm{E}-($ & $-2.06 \mathrm{E}-02$ & E-01 & -2 & $-2.25 E-01$ & -2 & -2.23 & $-2.26 \mathrm{E}-01$ \\
\hline$A_{1}$ & $-2.43 \mathrm{E}-04$ & $-2.57 \mathrm{E}-04$ & $-1.20 \mathrm{E}-03$ & $-1.22 \mathrm{E}-03$ & $-1.21 \mathrm{E}-03$ & -1.20 & $-1.09 \mathrm{I}$ & $-1.09 \mathrm{E}-03$ \\
\hline$A_{2}$ & $5.53 \mathrm{E}-06$ & $6.02 \mathrm{E}-06$ & $7.32 \mathrm{E}-05$ & $7.56 \mathrm{E}-05$ & $5.00 \mathrm{E}-05$ & -05 & 4.4 & 4.49E-05 \\
\hline$A_{3}$ & $-3.06 \mathrm{E}-08$ & -3.63 & $-1.02 \mathrm{E}-06$ & -1.1 & 2.7 & 3. & 6.5 & $7.26 \mathrm{E}-08$ \\
\hline$B_{1}$ & $4.11 \mathrm{E}-05$ & $3.42 \mathrm{E}-05$ & $1.34 \mathrm{E}-04$ & $1.38 \mathrm{E}-04$ & $6.79 \mathrm{E}-05$ & $3.31 \mathrm{E}-05$ & $2.17 \mathrm{E}-04$ & $2.14 \mathrm{E}-04$ \\
\hline$B_{2}$ & $-4.82 \mathrm{E}-05$ & $-5.32 \mathrm{E}-05$ & $-1.53 \mathrm{E}-03$ & $-1.53 \mathrm{E}-03$ & $-1.30 \mathrm{E}-03$ & $-1.31 \mathrm{E}-03$ & $-1.77 \mathrm{E}-03$ & $-1.79 \mathrm{E}-03$ \\
\hline
\end{tabular}

Table 4. Calibrated parameter of all cameras with our method and the reference calibration in Aicon 3D Studio.

and $0.050 \mathrm{~mm}(\mathrm{Y})$. As can be expected the Z- direction is less accurate with a maximum deviation of $0.44 \mathrm{~mm}$ and a RMSE of $0.097 \mathrm{~mm}$. When positioning two components relative to each other or the point of interest is not surrounded with points the error will increase.

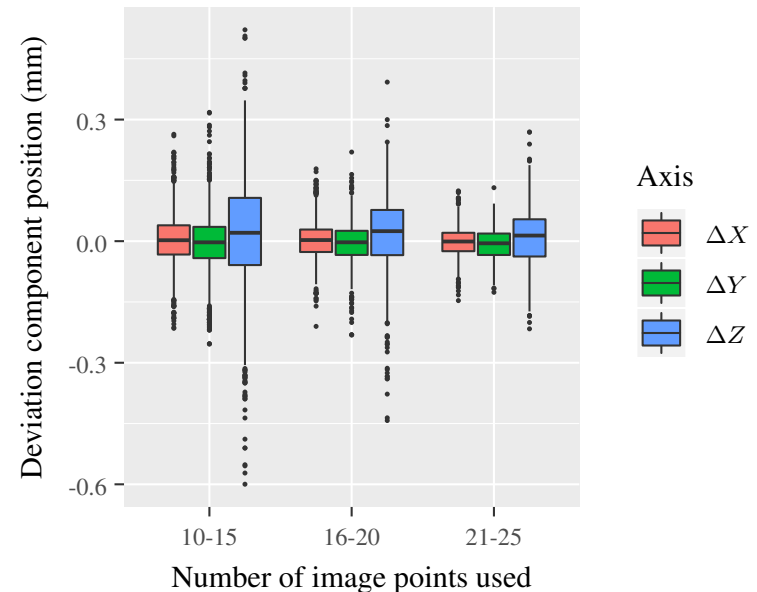

Figure 7. Deviations of position of one system component (in the camera coordinate system) using different numbers of observed image points in all four camera.

\section{CONCLUSION AND OUTLOOK}

The first comparison between the results of the two multi camera calibration methods shows that the implementation with Ceres is a usable choice for the measurement systems. Reason for the remaining systematic errors could not be determined and will be further investigated as soon the final prototype of the system is build. Scale measurements in the system are possible on sub-millimeter level only using the four images of one time step. The accuracy of determining the position of a system component, which is more relevant to the final measurement system is more accurate due to the use of more points. The achieved accuracy of $50 \mu \mathrm{m}(\mathrm{X}, \mathrm{Y})$ and $100 \mu \mathrm{m}$ in $\mathrm{Z}$ - direction will be enough for the extraction of samples from Legionella cultures.

The accuracy of final system depends on many factors that can not be tested jet. The camera positioning as well as the number and position of object points is not finally defined. Additionally will the image point accuracy be influenced by system lighting and spherical marker detection. Outlier detection and removal if necessary will also have an effect. But most important might be the overall stability of the relative orientation between the cameras while the system is running. Due to the limited number of markers on the system components and the resulting low number of observations the adjustment of the relative orientation during the operation will most likely not be an option.
Further investigations are necessary in many aspects. Possible changes of the principal distance and principal point during operation due to changing sensor temperature. Additional improvements of the calibration such as outlier detection or extended camera models for the reduction of systematic errors are useful. For the determination of the orientation of system components a stabilization over time using a Kalman-Filter might be reasonable to reduce the influence of noise. Beyond that many photogrammetric aspects of the measurement system have to be developed, like the position estimation of the cultures and the determination of the 3D geometry of the manipulation tools.

\section{ACKNOWLEDGEMENTS}

The Project 3Dbot is financed by the SAB with funds from the EFRE and tax money approved by the Saxon State Parliament.

\section{REFERENCES}

Agarwal, S., Mierle, K., Others, 2019. Ceres solver. http:// ceres-solver.org.

Bradski, G., 2000. The OpenCV Library. Dr. Dobb's Journal of Software Tools.

Brown, D. C., 1971. Close-range camera calibration. Photogramm. Eng, 37(8), 855-866.

Durovic, P., Grbic, R., Cupec, R., Filko, D., 2017. Low cost robot arm with visual guided positioning. 2017 40th International Convention on Information and Communication Technology, Electronics and Microelectronics (MIPRO), IEEE.

Hefele, J., Brenner, C., 2001. Robot pose correction using photogrammetric tracking. K. G. Harding, J. W. V. Miller, B. G. Batchelor (eds), Machine Vision and Three-Dimensional Imaging Systems for Inspection and Metrology, SPIE.

Huber, P. J., 1964. Robust Estimation of a Location Parameter. The Annals of Mathematical Statistics, 35(1), 73-101.

Luhmann, T., Fraser, C., Maas, H.-G., 2016. Sensor modelling and camera calibration for close-range photogrammetry. ISPRS Journal of Photogrammetry and Remote Sensing, 115, 37-46.

Oberthür, P., Otto, C., Lenk, F., 2016. Automatische Inspektion von Kulturschalen mit der PetriJet-Plattform. BIOspektrum, 22(5), 476-479.

Renaud, P., Andreff, N., Lavest, J.-M., Dhome, M., 2006. Simplifying the kinematic calibration of parallel mechanisms using vision-based metrology. IEEE Transactions on Robotics, 22(1), $12-22$. 
Sargeant, B., Robson, S., Szigeti, E., Richardson, P., El-Nounu, A., Rafla, M., 2016. A Method to Achieve Large Volume, High Accuracy Photogrammetric Measurements Through the Use of an Actively Deformable Sensor Mounting Platform. ISPRS International Archives of the Photogrammetry, Remote Sensing and Spatial Information Sciences, XLI-B5, 123-129.

Shu, T., Gharaaty, S., Xie, W., Joubair, A., Bonev, I. A., 2018. Dynamic Path Tracking of Industrial Robots With High Accuracy Using Photogrammetry Sensor. IEEE/ASME Transactions on Mechatronics, 23(3), 1159-1170.

Triggs, B., McLauchlan, P. F., Hartley, R. I., Fitzgibbon, A. W., 1999. Bundle adjustment - a modern synthesis. International workshop on vision algorithms, Springer, 298-372.

Vogel, M., Boschke, E., Bley, T., Lenk, F., 2015. PetriJet Platform Technology. Journal of Laboratory Automation, 20(4), 447-456.

Werner, T., Harrer, D., Henrich, D., 2018. Efficient, precise, and convenient calibration of multi-camera systems by robot automation. Advances in Service and Industrial Robotics, Springer International Publishing, 669-677.

Zhang, X., Song, Y., Yang, Y., Pan, H., 2017. Stereo vision based autonomous robot calibration. Robotics and Autonomous Systems, 93, 43-51. 